\title{
Índices periódicos por volumen
}

Vol. 1, Núm. 2 (2003)

Tabla de contenidos

\section{Artículos originales}

Investigación y proyecto arquitectónico

Autor: Susana Jiménez Correa

APA: Jiménez-Correa, S. (2003). Investigación y proyecto arquitectónico. Revista Científica Guillermo de Ockham, 1(2), 13-40.

Los procesos de apropiación del conocimiento arquitectónico. Un análisis interpretativo

Autor: Germán Darío Correal Pachón

APA: Correal-Pachón, G. D. (2003). Investigación y proyecto arquitectónico. Revista Científica Guillermo de Ockham, 1(2), 41-56.

Retórica y socialización en tres generaciones

Autores: José Fernando Ossa Ramírez, Elsy González Velásquez, Luz Estela Rebelo Quirama

APA: Ossa-Ramírez, J. F., González-Velásquez, E., \& Rebelo-Quirama, L. E. (2003). Retórica y socialización en tres generaciones. Revista Científica Guillermo de Ockham, 1(2), 57-87.

Ley Páez en el norte del Cauca, Colombia, y su influencia sobre la comunidad de Villarica. Hallazgos iniciales

Autores: Verena González Cabo, Alvaro Valencia Orozco

APA: Verena-González, C., \& Valencia-Orozco, A. (2003). Ley Páez en el norte del Cauca, Colombia, y su influencia sobre la comunidad de Villarica. Hallazgosiniciales. Revista Científica Guillermo de Ockham, 1(2), 88-100.

Agroindustria en el norte del Cauca: una mirada histórica. Informe preliminar de investigación

Autor: HernánZuluaga Albarracín

APA: Zuluaga-Albarracín, H. (2003). Agroindustria en el norte del Cauca: una mirada histórica. Informe preliminar de investigación. Revista Científica Guillermo de Ockham, 1(2), 101-111.

El trabajo: fin, crisis o redefinición

Autor: Carlos Augusto Patiño Uribe

APA: Patiño-Uribe, C. A. (2003). El trabajo: fin, crisis o redefinición. Revista Científica

Guillermo de Ockham, 1(2), 112-129.

Una mirada aproximativa a la educación superior en España, Alemania y Francia

Autor: María Eugenia Salinas Muñoz

APA: Salinas-Muñoz, M. E. (2003). Una mirada aproximativa a la educación superior en España, Alemania y Francia. Revista Científica Guillermo de Ockham, 1(2), 130-165.

Franciscanismo en América: Un acercamiento a la experiencia mexica. El "Diálogo de los Doce"

Autor: Antonio José Echeverry Pérez

APA: Echeverry-Pérez, A. J. (2003). Franciscanismo en América: Un acercamiento a la experiencia mexica. El "Diálogo de los Doce". Revista Científica Guillermo de Ockham, 1(2), 166-175.

Crónicas e imaginario de la Conquista

Autor: Johannio Marulanda Arbeláez 
APA: Marulanda-Arbeláez, J. (2003). Crónicas e imaginario de la Conquista. Revista Cientifica Guillermo de Ockham, 1(2), 176-187.

\section{Vol. 2, Núm. 1 (2004)}

Tabla de contenidos

\section{Artículos originales}

Conflicto y violencia escolar en Colombia Lectura breve de algunos materiales escritos

Autor: Fernando Valencia Murcia

APA: Valencia-Murcia, F. (2004). Conflicto y violencia escolar en Colombia Lectura breve de algunos materiales escritos. Revista Cientifica Guillermo de Ockham, 2(1), 29-41.

La flexibilidad: eje de una estructura académico-administrativa

Autor: Orfa Garzón Rayo

APA: Garzón-Rayo, O. (2004). La flexibilidad: eje de una estructura académicoadministrativa. Revista Científica Guillermo de Ockham, 2(1), 11-28.

Cuerpo, prácticas sociales y modernidad. Tecnologías y representaciones de la corporalidad en la transformación europea, siglos XVI al XVIII

Autor: José Luis Grosso Lorenzo

APA: Grosso-Lorenzo, J. L. (2004). Cuerpo, prácticas sociales y modernidad. Tecnologías y representaciones de la corporalidad en la transformación europea, siglos XVI al XVIII . Revista Científica Guillermo de Ockham, 2(1), 43-58.

El proyecto arquitectónico: campo de conocimiento y realización Entrevista a Rogelio Salmona

Autor: Susana Jiménez Correa

APA: Jiménez-Correa, S. (2004). Cuerpo, prácticas sociales y modernidad. Tecnologías y representaciones de la corporalidad en la transformación europea, siglos XVI al XVIII.

Revista Científica Guillermo de Ockham, 2(1),

Sobre la naturaleza de la investigación proyectual

Autor: Germán Darío Correal Pachón

APA: Correal-Pachón, G. D. (2004). Sobre la naturaleza de la investigación proyectual. Revista Científica Guillermo de Ockham, 2(1), 91-111.

¿Son los sumideros una nueva forma de cooperación internacional para el desarrollo?

Autor: Carlos Alfredo Matallana Martínez

APA: Matallana-Martínez, C. A. (2004). ¿Son los sumideros una nueva forma de cooperación internacional para el desarrollo?. Revista Científica Guillermo de Ockham, 2(1), 139-185.

Efecto insecticida y residual de tres extractos de Lippia alba para el control de Acanthoscelides obtectus en fríjol Diacol Calima

Autores: Sandra Patricia Guzmán Rivera, Adolfo Truchez Parra, Luis Fernando Correa Zapata, Mónica Zuñiga López

APA: Guzmán-Rivera, S. P., Truchez-Parra, A., Correa-Zapata, L. F., \& Zuñiga-López, M. (2004). Efecto insecticida y residual de tres extractos de Lippia alba para el control de Acanthoscelides obtectus en fríjol Diacol Calima. Revista Científica Guillermo de Ockham, 2(1), 187-199.

Desarrollo agrotecnológico de Lippia alba (Miller) N. E. Brown ex Britton \& Wilson

Autores: Sandra Patricia Guzmán Rivera, Ricardo Cardozo, Vanessa García 
APA: Guzmán-Rivera, S. P., \& Cardozo, R. (2004). Desarrollo agrotecnológico de Lippia alba (Miller) N. E. Brown ex Britton \& Wilson. Revista Científica Guillermo de Ockham, 2(1), 201215.

Greens function formalism for the study of the role of $2 \mathrm{~d}$-magnetoplasmons on magneto infra-red absorption in high electronic density quantum wells

Autor: Gonzalo Becerra Orozco

APA: Becerra-Orozco, G. (2004). Greensfunction formalism for the study of the role of $2 d$ magnetoplasmons on magneto infra-red absorption in high electronic density quantum wells. Revista Científica Guillermo de Ockham, 2(1), 217-222.

\section{Vol. 2, Núm. 2 (2004)}

Tabla de contenidos

\section{Artículos originales}

La actividad empresarial desarrollada por la comunidad de Villarica, Cauca Acontecimientos sociales, económicos y políticos sucedidos a partir de 1970

Autor: José Alonso González Solano

APA: González-Solano, J. A. (2004). La actividad empresarial desarrollada por la comunidad de Villarrica, Cauca Acontecimientos sociales, económicos y políticos sucedidos a partir de 1970. Revista Científica Guillermo de Ockham, 2(2), 15-43.

Las formas empresariales de Villarica durante la primera administración municipal El desarrollo empresarial del municipio, antes y después de la primera alcaldía elegida por voto popular

Autor: Verena González Cabo

APA: González-Cabo, V. (2004). La actividad empresarial desarrollada por la comunidad de Villarrica, Cauca Acontecimientos sociales, económicos y políticos sucedidos a partir de 1970. Revista Científica Guillermo de Ockham, 2(2), 45-57.

Gestión pública canadiense Vs. gestión pública colombiana: el manejo de las finanzas públicas

Autor: Rosa Evelia Sánchez

APA: Sánchez, R. E. (2004). Gestión pública canadiense Vs. gestión pública colombiana: el manejo de las finanzas públicas. Revista Científica Guillermo de Ockham, 2(2), 59-96.

Del conflicto social al conflicto moral Aproximación a una posición política y jurídica del reconocimiento de la identidad

Autor: Luis Fredyur Tovar

APA: Freydur-Tovar, L. (2004). Del conflicto social al conflicto moral Aproximación a una posición política y jurídica del reconocimiento de la identidad. Revista Científica Guillermo de Ockham, 2(2), 97-108.

Apuntes sobre formación política: responsabilidad ciudadana

Autor: Leonardo Rojas

APA: Rojas, L. (2004). Apuntes sobre formación política: responsabilidad ciudadana. Revista Científica Guillermo de Ockham, 2(2), 109-138.

El movimiento indígena y sus paradigmas de interpretación

Autor: Nidia Catherine González Piñeres

APA: González-Piñeres, N. C. (2004). El movimiento indígena y sus paradigmas de 
interpretación. Revista Científica Guillermo de Ockham, 2(2),

Memoria y recuerdo colectivos Algunas consideraciones sobre el caso de una leyenda en Mulaló (Valle del Cauca - Colombia)

Autor: Fernando Valencia Murcia

APA: Valencia-Murcia, F. (2004). Memoria y recuerdo colectivos Algunas consideraciones sobre el caso de una leyenda en Mulaló (Valle del Cauca - Colombia). Revista Científica Guillermo de Ockham, 2(2), 163-185.

La creatividad como estrategia de intervención educativa en los conflictos escolares. El caso del Centro de Desarrollo Comunitario de Siloé

Autor: Constanza Moncada Galvis

APA: Moncada-Galvis, C. (2004). La creatividad como estrategia de intervención educativa en los conflictos escolares. El caso del Centro de Desarrollo Comunitario de Siloé. Revista Cientifica Guillermo de Ockham, 2(2), 187-201.

\section{Vol. 3, Núm. 1 (2005)}

Tabla de contenidos

\section{Artículos originales}

Procesos cognitivos en la proyectación arquitectónica. Análisis de un ejercicio

Autor: Germán Darío Correal Pachón

APA: Correal-Pachón G. D. (2005). Procesos cognitivos en la proyectación arquitectónica. Análisis de un ejercicio. Revista Científica Guillermo de Ockham, 3(1), 9-26.

Los conceptos de bienestar y satisfacción. Una revisión de tema

Autor: José Fernando Ossa

APA: Ossa, J. F. (2005). Los conceptos de bienestar y satisfacción. Una revisión de tema. Revista Científica Guillermo de Ockham, 3(1), 27-59.

Dialéctica de las utopías: Del no lugar al lugar perfecto en el imaginario franciscano. 1550 - 1630

Autor: Antonio José Echeverry Pérez

APA: Echeverry-Pérez, A. J. (2005). Dialéctica de las utopías: Del no lugar al lugar perfecto en el imaginario franciscano. 1550 - 1630. Revista Científica Guillermo de Ockham, 3(1), 61-81.

Prácticasy herramientas de desarrollo utilizadas en empresas de Cali

Autor: Jaime Alberto Chavarrriaga

APA: Chavarrriaga, J. A. (2005). Prácticas y herramientas de desarrollo utilizadas en empresas de Cali. Revista Científica Guillermo de Ockham, 3(1), 83-91.

Dispersión Random Walk, irreversibilidad y velocidad del flujo no uniforme en los cauces turbulentos

Autor: Alfredo Constaín Aragón

APA: Constaín-Aragón A. C. (2005). Dispersión Random Walk, irreversibilidad y velocidad del flujo no uniforme en los cauces turbulentos. Revista Científica Guillermo de Ockham, 3(1), 93-114. 


\section{Vol. 4, Núm. 2 (2006)}

Tabla de contenidos

\section{Artículos originales}

Resurgir de las utopías desde un paradigma franciscano

Autor: Antonio José EcheverriPérez

APA: Echeverri-Pérez A. J. (2006). Resurgir de las utopías desde un paradigma franciscano. Revista Científica Guillermo de Ockham, 4(2), 53-70.

Ayuda mutua e intercambio: hacia una aproximación conceptual

Autor: Fernando Valencia Murcia

APA: Valencia-Murcia, F. (2006). Ayuda mutua e intercambio: hacia una aproximación conceptual. Revista Científica Guillermo de Ockham, 4(2), 71-81.

Valoración histórica del contexto construido. Cali, una mirada local

Autor: Susana Jiménez Correa

APA: Jiménez-Correa, S. (2006). Valoración histórica del contexto construido. Cali, una mirada local. Revista Científica Guillermo de Ockham, 4(2), 83-120.

Hacia una cultura de emprendimiento productivo en comunidad

Autor: José Alonso González

APA: González, J. A. (2006). Hacia una cultura de emprendimiento productivo en comunidad. Revista Científica Guillermo de Ockham, 4(2), 133-153.

Enseñanzas del proceso de erradicación de asentamientos marginales en Córdoba, Argentina

Autor: Olga Lucía Montoya Flórez

APA: Montoya-Flórez, O. L. (2006). Enseñanzas del proceso de erradicación de asentamientos marginales en Córdoba, Argentina. Revista Científica Guillermo de Ockham, 4(2), 121-132.

Una mirada al conflicto escolar desde el maestro y su quehacer cotidiano

Autor: Lorena Arango Quiroz

APA: Arango-Quiroz, L. (2006). Una mirada al conflicto escolar desde el maestro y su quehacer cotidiano. Revista Científica Guillermo de Ockham, 4(2), 155-170.

\section{Vol. 5, Núm. 1 (2007)}

Tabla de contenidos

\section{Artículos originales}

La medicina tradicional y el problema de la salud: Inicio de un recorrido en el estudio de la salud mental

Autor: John James Gómez Gallego

APA: Gómez-Gallego, J. J. (2007). La medicina tradicional y el problema de la salud: Inicio de un recorrido en el estudio de la salud mental. Revista Científica Guillermo de Ockham, 5(1), 13-28. 
La contextualización del currículo: Cognición y no verbalidad

Autor: Claudia Mallarino Flórez

APA: Mallarino-Flórez, C. (2007). La contextualización del currículo: Cognición y no verbalidad. Revista Científica Guillermo de Ockham, 5(1), 73-84.

Gente bien, gente decente: Fronteras simbólicas, procesos de identificación y diferenciación de profesionales pertenecientes a las clases medias en Cali

Autor: José Fernando Sánchez Salcedo

APA: Sánchez-Salcedo, J. F. (2007). Gente bien, gente decente: Fronteras simbólicas, procesos de identificación y diferenciación de profesionales pertenecientes a las clases medias en Cali. Revista Científica Guillermo de Ockham, 5(1), 85-100.

La transmisión de la moral cristiana desde el lenguaje religioso

Autor: Leonardo Rojas Cadena

APA: Rojas-Cadena, L. (2007). La transmisión de la moral cristiana desde el lenguaje religioso. Revista Científica Guillermo de Ockham, 5(1), 101-113.

\section{Fronteras:}

Leebeus Woods

Autor: Armando Buchard de la $\mathrm{Hoz}$

APA: Buchard de la Hoz, A. (2007). Leebeus Woods. Revista Científica Guillermo de Ockham, 5(1), 117-120.

\section{Reseñas:}

Libro: El conquistador

Autor: John James Gómez

APA: Gómez, J. J. (2007). Reseña del libro El conquistador, de F. Andahazi. Revista Científica Guillermo de Ockham, 5(1), 123-124.

Libro: El viajero en el Umbral

Autor: César Mejía

APA: Mejía, C. (2007). Reseña del libro El viajero en el Umbral, de G. J. Alzate. Revista Científica Guillermo de Ockham, 5(1), 125-126.

\section{Vol. 5, Núm. 2 (2007)}

Tabla de contenidos

\section{Artículos originales}

¿Cómo se entiende formar para la investigación en contextos de educación media y de educación superior?

Autor: Laura María Pineda Villany, Lorena Uribe, Emiliano Díaz

APA: Pineda-Villany, L. M., Uribe, L., \& Díaz, E. (2007). ¿Cómo se entiende formar para la investigación en contextos de educación media y de educación superior?. Revista Científica Guillermo de Ockham, 5(2), 11-22.

Alternativas de identificación. Una revisión de tema 
Autor: José Fernando Ossa Ramírez

APA: Ossa-Ramírez, J. F. (2007). Alternativas de identificación. Una revisión de tema.

Revista Cientifica Guillermo de Ockham, 5(2), 23-52.

Narrativas docentes y experiencias escolares significativas: relatando el sentido de ser maestro

Autor: Cristhian James Díaz Meza

APA: Díaz-Meza, C. J. (2007). Narrativas docentes y experiencias escolares significativas: relatando el sentido de ser maestro. Revista Científica Guillermo de Ockham, 5(2), 55-65.

Modernidad y sentido Una exigencia cultural para la teología

Autor: Leonardo Rojas Cadena

APA: Rojas-Cadena, L. (2007). Modernidad y sentido Una exigencia cultural para la teología. Revista Científica Guillermo de Ockham, 5(2), 67-81.

Trayectoria de las empresas multinacionales en la ciudad de Cali

Autores: Johnny Javier Orejuela, Camilo Mayor, Juan Javier Vesga

APA: Orejuela, J. J., Mayor, C., \& Vesga, J. J. (2007). Modernidad y sentido Una exigencia cultural para la teología. Revista Científica Guillermo de Ockham, 5(2), 83-103.

Fronteras de oportunidad. El proyecto desde la lógica ambiental

Autor: Mariela Marchisio

APA: Marchisio, M. (2007). Fronteras de oportunidad. El proyecto desde la lógica ambiental. Revista Científica Guillermo de Ockham, 5(2), 105-109.

Señales visuales urbanas en algunos cruces viales de Cali, Colombia

Autores: Marco Alexis Salcedo, Silvia Cristina Caicedo

APA: Salcedo, M. A., \& Caicedo, S. C. (2007). Señales visuales urbanas en algunos cruces viales de Cali, Colombia. Revista Científica Guillermo de Ockham, 5(2), 111-133.

\section{Fronteras:}

Semillero culturas juveniles y contemporaneidad: un escenario intersticial para la formación investigativa.

Autor: José Fernando Patiño

APA: Patiño, J. F. (2007). Semillero culturas juveniles y contemporaneidad: un escenario intersticial para la formación investigativa. Revista Científica Guillermo de Ockham, 5(2), 137-141.

\section{Semblanza:}

Entrevista a Nestor Braunstein

Autores: John James Gómez y Johnny Javier Orejuela

APA: Gómez, J. J., \& Orejuela, J. J. (2007).Entrevista a Néstor Braunstein. Revista Científica Guillermo de Ockham, 5(2), 143-154.

\section{Reseñas:}

Libro: Análisis de la producción y las operaciones

Autor: Luis Alfredo EcheverriP. 
APA: Echeverri, L. A. (2007). Reseña del libro Análisis de la producción y las operaciones, de S. Nahamias. Revista Científica Guillermo de Ockham, 5(2), 157-158.

Libro: Los niños de nadie. Trata de personas a ras de asfalto

Autor: Dulfay Astrid González

APA: González, D. A. (2007). Reseña del libro Los niños de nadie. Trata de personas a ras de asfalto, de V. Ronquillo. Revista Científica Guillermo de Ockham, 5(2), 159-160.

Libro: Memorias de la salsa

Autor: Israel Sánchez Coll

APA: Sánchez-Coll, I. (2007). Reseña del libro Memorias de la salsa. Trata de personas a ras de asfalto, de W. Magaña. Revista Científica Guillermo de Ockham, 5(2), 161-162.

\section{Vol. 6, Núm. 1 (2008)}

Tabla de contenidos

\section{Artículos originales}

La observación, una palabra para desbaratar y re-significar

Autor: Rafael Ávila

APA: Ávila, R. (2008). La observación, una palabra para desbaratar y re-significar. Revista Científica Guillermo de Ockham, 6(1), 15-26.

El quehacer del psicólogo en el trabajo con comunidades tradicionales

Autor: John James Gómez Gallego

APA: Gómez-Gallego, J. J. (2008). El quehacer del psicólogo en el trabajo con comunidades tradicionales. Revista Científica Guillermo de Ockham, 6(1), 27-35.

Definición de una metodología ágil de ingeniería de requerimientos para empresas emergentes de desarrollo de software del sur-occidente colombiano

Autores: Luis Merchán, Alba Urrea, Rubén Rebollar

APA: Merchán, L., Urrea, A., \& Rebollar, R. (2008). Definición de una metodología ágil de ingeniería de requerimientos para empresas emergentes de desarrollo de software del sur-occidente colombiano. Revista Cientifica Guillermo de Ockham, 6(1), 37-50.

Didáctica en la condición postmoderna. De las competencias a la cooperación

Autores: Germán Vargas Guillén, Sonia Cristina Gamboa Sarmiento

APA: Vargas-Guillén, G., \& Gamboa-Sarmiento, S. C. (2008). Didáctica en la condición postmoderna. De las competencias a la cooperación. Revista Científica Guillermo de Ockham, 6(1), 51-59.

Imaginarios y utopía franciscana en Nueva Granada en el siglo XVI

Autores: Antonio José Echeverri Pérez, Johannio Marulanda Arbeláez

APA: Echeverri-Pérez, A. J., \& Marulanda-Arbeláez, J. (2008). Imaginarios y utopía franciscana en Nueva Granada en el siglo XVI. Revista Científica Guillermo de Ockham, 6(1), 61-77.

Aproximaciones a la flexibilidad curricular en la educación superior en Colombia 
Autor: Patricia Medina Agredo

APA: Medina-Agredo, P. (2008). Aproximaciones a la flexibilidad curricular en la educación superior en Colombia. Revista Científica Guillermo de Ockham, 6(1), 79-89.

Determinación de la factibilidad del hongo Metarhizium anisopliae para ser usado como control biológico de la hormiga arriera (Atta cephalotes)

Autor: Yuly Lemus, Ginna Rodríguez, Raúl Cuervo, Jorge Antonio Durán Vanegas, Claudia Liliana Zuluaga

APA: Lemus, Y., Rodríguez, G., Cuervo, R., Durán, J. A., \& Zuluaga, C. L. (2008).

Determinación de la factibilidad del hongo Metarhizium anisopliae para ser usado como control biológico de la hormiga arriera (Atta cephalotes). Revista Científica Guillermo de Ockham, 6(1), 91-98.

El espacio público como objeto de estudio en las ciencias sociales y humanas

Autores: Marco Alexis Salcedo, Silvia Cristina Caicedo

APA: Salcedo, M. A., \& Caicedo, S. C. (2008). El espacio público como objeto de estudio en las ciencias sociales y humanas. Revista Científica Guillermo de Ockham, 6(1), 99-115.

\section{Fronteras}

Autor: Claudia Mallarino López

APA: Mallarino-López, C. (2008). La danza contemporánea en el transmilenio. Revista Científica Guillermo de Ockham, 6(1), 119-125.

\section{Semblanzas}

Entrevista a la profesora Flor Alba Cano

Autor: Johnny Orejuela

APA: Orejuela, J. (2008). Entrevista a la profesora Flor Alba Cano. Revista Científica Guillermo de Ockham, 6(1), 129-142.

\section{Reseñas}

Libro: Desarrollo empresarial y emprendimiento en Colombia informe del estudio GEM Colombia 2006

Autor: José Alonso González

APA: Gónzalez, J. A. (2008). Reseña del libro Desarrollo empresarialy emprendimiento en Colombia, de informe del estudio GEM Colombia 2006. Revista Científica Guillermo de Ockham, 6(1), 145-148. 


\section{Vol. 6, Núm. 2 (2008)}

Tabla de contenidos

Artículos originales

Estado actual de las investigaciones sobre mujer casada, profesional y madre: del trabajo remunerado al trabajo doméstico

Autor: María Fernanda Rodríguez Montaño

APA: Rodríguez-Montaño, M. F. (2008). Estado actual de las investigaciones sobre mujer casada, profesional y madre: del trabajo remunerado al trabajo doméstico. Revista Cientifica Guillermo de Ockham, 6(2), 13-25.

La teología moral hoy: sus desafíos

Autor: Diego Agudelo Grajales

APA: Agudelo-Grajales, D. (2008). La teología moral hoy: sus desafíos. Revista Científica Guillermo de Ockham, 6(2), 27-36.

Alineación de los programas de capacitación con los procesos de innovación en pymes del sector confecciones del Valle del Cauca

Autores: Armando Mejía Giraldo, Mario Bravo Castillo

APA: Mejía-Giraldo, A., \& Bravo-Castillo, M. (2008). Alineación de los programas de capacitación con los procesos de innovación en pymes del sector confecciones del Valle del Cauca. Revista Científica Guillermo de Ockham, 6(2), 37-53.

La didáctica: acontecimiento vivo en el aula

Autor: Beatriz Lorena Buitrago

APA: Buitrago, B. L. (2008). La didáctica: acontecimiento vivo en el aula. Revista Científica Guillermo de Ockham, 6(2), 55-67.

Trayectorias laborales y relacionales de los profesionales insertos en mercadosglobales a través de empresas multinacionales (EMN) con presencia en la ciudad de Cali, Colombia

Autor: Johnny Javier Orejuela

APA: Orejuela, J. J. (2008). Trayectorias laborales y relacionales de los profesionales insertos en mercados globales a través de empresas multinacionales (EMN) con presencia en la ciudad de Cali, Colombia. Revista Científica Guillermo de Ockham, 6(2), 69-80.

Descubrimiento automático de equivalencias en esquemas de bases de datos relacionales

Autor: Christian Arias

APA: Arias, C. (2008). Descubrimiento automático de equivalencias en esquemas de bases de datos relacionales. Revista Cientifica Guillermo de Ockham, 6(2), 81-89.

Una reflexión crítica sobre ciudad educadora

Autor: Marco Alexis Salcedo

APA: Salcedo, M. A. (2008). Una reflexión crítica sobre ciudad educadora. Revista Científica Guillermo de Ockham, 6(2), 91-107.

El nuevo itinerario de la Iglesia es la comunión y la solidaridad

Autores: Jesús Carrasquilla, Leonardo Rojas

APA: Carrasquilla, J., \& Rojas, L. (2008). El nuevo itinerario de la Iglesia es la comunión y la solidaridad. Revista Científica Guillermo de Ockham, 6(2), 109-120. 


\section{Fronteras}

Jaime Henao. Contrapunto y disonancia

Autor: César Mejía y Alexander Aguirre

APA: Mejía, C. , \& Aguirre, A. (2008). Jaime Henao. Contrapunto y disonancia. Revista Científica Guillermo de Ockham, 6(2), 123-133.

\section{Reseñas}

Libro: Clientelismo y fe: dinámicas políticas

Autor: Luis Eduardo Rojas

APA: Rojas, L. E. (2008). Reseña del libro Clientelismo y fe: dinámicas políticas, de A. Cepeda-van Houten. Revista Científica Guillermo de Ockham, 6(2), 137-138.

Libro: Recensión: justos por pecadores oculta del Opus Dei

Autor: Carolino Romero Burbano

APA: Romero-Burbano, C. (2008). Reseña del libro Recensión: justos por pecadores oculta del Opus Dei, de F. Quiroz. Revista Científica Guillermo de Ockham, 6(2), 139-140.

\section{Vol. 7, Núm. 1 (2009)}

Tabla de contenidos

\section{Artículos originales}

Efecto de las concentraciones salinas en la inhibición de Leuconostoc mesenteroides en un ingenio azucarero del Valle del Cauca

Autores: Johany Ángel, Raúl Cuervo Mulet, Heiber Cárdenas, Jorge Antonio Durán, Luis Fernando Mejía, Gloria Rodríguez de la Pava.

APA: Ángel et al. (2009). Efecto de las concentraciones salinas en la inhibición de Leuconostoc mesenteroides en un ingenio azucarero del Valle del Cauca. Revista Científica Guillermo de Ockham, 7(1), 13-18.

Mentes, videojuegos y sociedad

Autores: César Mejía, Manuela Rodríguez Mora, Beatriz Castellanos Jaramillo APA: Mejía, C., Rodríguez-Mora, M., \& Castellanos Jaramillo, B. C. (2009). Efecto de las concentraciones salinas en la inhibición de Leuconostoc mesenteroides en un ingenio azucarero del Valle del Cauca. Revista Científica Guillermo de Ockham, 7(1), 19-30.

Educación superior latinoamericana. Factores claves que tipifican la educación superior en América Latina hoy

Autor: Martha Lucía Ramírez Pérez

APA: Ramírez Pérez, M. L. (2009). Educación superior latinoamericana. Factores claves que tipifican la educación superior en América Latina hoy. Revista Científica Guillermo de Ockham, 7(1), 31-39. 
Primero afirmar, luego integrar. La interdisciplinariedad y las ciencias sociales

Autor: Johnny Javier Orejuela

APA: Orejuela, J. J. (2009). Primero afirmar, luego integrar. La interdisciplinariedad y las ciencias sociales. Revista Científica Guillermo de Ockham, 7(1), 41-56.

Desplazamiento forzado en Florida, Valle del Cauca. Mujeres, territorio y cultura

Autor: Rubén Darío Guevara, Felipe Barney Arango

APA: Guevara, R. D., \& Barney-Arango, F. Ossa Ramírez

Producción discursiva de vivencias de bienestar mediante retórica y géneros discursivos cotidianos

Autor: José Fernando Ossa Ramírez

APA: Ossa-Ramírez, J. F. (2009). Primero afirmar, luego integrar. La interdisciplinariedad y las ciencias sociales. Revista Científica Guillermo de Ockham, 7(1), 69-82.

Panorama de las estéticas contemporáneas en la arquitectura local

Autor: Armando Buchard de la $\mathrm{Hoz}$

APA: Buchard de la Hoz, A. (2009). Primero afirmar, luego integrar. La

interdisciplinariedad y las ciencias sociales. Revista Científica Guillermo de Ockham, 7(1), 83-104.

La siembra: de tierra, polvo y otras vicisitudes

Autor: María Elena Díaz Rico, Nancy Faride Arias, Patricia Lasso Toro

APA: Díaz-Rico, M. E., Faride-Arias, N., \& Lasso-Toro, P. (2009). La siembra: de tierra, polvo y otras vicisitudes. La interdisciplinariedad y las ciencias sociales. Revista Cientifica Guillermo de Ockham, 7(1), 105-122.

Voluntad y conocimiento como presupuestos del dolo en materia penal

Autor: Yesid Echeverry Enciso

APA: Echeverry-Enciso, Y. (2009). Voluntad y conocimiento como presupuestos del dolo en materia penal. Revista Científica Guillermo de Ockham, 7(1), 123-151.

\section{Fronteras}

Araceli psicoanalizada. Sobre la novela Araceli, de Elsa Morante

Autor: Alfonso Rubio Hernández

APA: Rubio-Hernández, A. (2009). Araceli psicoanalizada. Sobre la novela Araceli, de Elsa Morante. Revista Científica Guillermo de Ockham, 7(1), 155-161.

\section{Semblanza}

Guillermo de Ockham (1285-1347/49) Francisco-filósofo-teólogo-político

Autor: Edgar Alonso Vanegas Carvajal

APA: Vanegas-Carvajal, E. A. (2009). Guillermo de Ockham (1285-1347/49) Franciscofilósofo-teólogo-político. Revista Científica Guillermo de Ockham, 7(1), 165-179. 


\section{Vol. 7, Núm. 2 (2009)}

Tabla de contenidos

Artículos originales

Síntesis, caracterización estructural y eléctrica de películas de V2O5. nH2O

Autores: César Leandro Londoño, Carlos Vargas Hernández, Fabián Jurado

APA: Londoño, C. L., Vargas-Hernández, C., \& Jurado, F. (2009). Síntesis, caracterización estructural y eléctrica de películas de V2O5. $\mathrm{nH} 2 \mathrm{O}$. Revista Científica Guillermo de Ockham, 7(2), 11-18.

Diseño y validación de un instrumento para evaluar limitaciones en la actividad y restricciones en la participación de los ancianos

Autor: Giovana Marcella Rosas Estrada

APA: Rosas-Estrada, G. M. (2009). Diseño y validación de un instrumento para evaluar limitaciones en la actividad y restricciones en la participación de los ancianos. Revista Científica Guillermo de Ockham, 7(2), 11-18.

Epistemología y didáctica del proceso de enseñanza aprendizaje en arquitectura y diseño industrial

Autor: Jemay Mosquera Téllez

APA: Mosquera-Téllez, J. (2009). Diseño y validación de un instrumento para evaluar limitaciones en la actividad y restricciones en la participación de los ancianos. Revista Científica Guillermo de Ockham, 7(2), 11-18.

Hidrólisis y fermentación alcohólica simultánea (HFS) del residuo agroindustrial del mango común (Mangifera indica L) utilizando levaduras Saccharomyces cerevisiae spp y cepa recombinante $\mathrm{RH} 218$

Autores: Luis Fernando Mejía, Diana Carolina Albán, Natalia Murcia, Raúl Cuervo, Jorge Durán

APA: Mejía, L. F., Albán, D. C., Murcia, N., Cuervo, R., \& Durán, J. (2009). Hidrólisis y fermentación alcohólica simultánea (HFS) del residuo agroindustrial del mango común (Mangifera indica L) utilizando levaduras Saccharomyces cerevisiae spp y cepa recombinante RH 218. Revista Científica Guillermo de Ockham, 7(2), 51-64.

Consideraciones sobre el paso a la vida civil de jóvenes desvinculados de grupos armados ilegales

Autor: Manuel Alejandro Moreno Camacho

APA: Moreno-Camacho, M. A. (2009). Consideraciones sobre el paso a la vida civil de jóvenes desvinculados de grupos armados ilegales. Revista Científica Guillermo de Ockham, 7(2), 65-74.

La juventud: una construcción social-histórica de Occidente

Autor: José Fernando Patiño Torres

APA: Patiño-Torres, J. F. (2009). La juventud: una construcción social-histórica de Occidente. Revista Científica Guillermo de Ockham, 7(2), 75-90.

Revisión de técnicas de análisis de decisión multicriterio (múltiple criteria decisión analysis MCDA) como soporte a problemas complejos: pronósticos de demanda 
Autor: Mario Fernando Acosta Ríos, Raúl Antonio Díaz Pacheco, Ángela Patricia Anaya Salazar

APA: Acosta-Ríos, M. F. (2009). Revisión de técnicas de análisis de decisión multicriterio (múltiple criteria decisión analysis -MCDA) como soporte a problemas complejos: pronósticos de demanda. Revista Científica Guillermo de Ockham, 7(2), 91110.

Desde fuera y desde dentro: aproximación preliminar a los estudios sobre identidad cultural en afrocolombianos

Autor: María Elena Díaz Rico

APA: Díaz-Rico, M. E. (2009). Desde fuera y desde dentro: aproximación preliminar a los estudios sobre identidad cultural en afrocolombianos. Revista Científica Guillermo de Ockham, 7(2), 111-132.

Nuestras ciudades y arquitectura

Autor: Benjamín Barney Caldas

APA: Barney-Caldas, B. (2009). Nuestras ciudades y arquitectura. Revista Científica Guillermo de Ockham, 7(2), 133-151.

\section{Reseñas:}

Estudiando la homosexualidad: Teoría e investigación

Autor: Octavio Orozco García

APA: Orozco-García, O. (2009). Estudiando la homosexualidad: Teoría e investigación, de J. I. Baile-Ayensa. Revista Científica Guillermo de Ockham, 7(2), 155-158.

\section{Fronteras}

Entrevista a Jean Allouch

Autores: Johnny Orejuela y Vanessa Salazar

APA: Orejuela, J., \& Salazar, V. (2009). Entrevista a Jean Allouch. Revista Cientifica Guillermo de Ockham, 7(2), 161-167.

\section{Vol. 8, Núm. 1 (2010)}

Tabla de contenidos

\section{Artículos originales}

Diagnóstico sobre la atención a la mujer víctima de violencia de género en la ciudad de Santiago de Cali

Autor: Diana Marcela Bustamante Arango, Milton Puertas

APA: Bustamante-Arango, D. M., \& Puertas, M. (2010). Diagnóstico sobre la atención a la mujer víctima de violencia de género en la ciudad de Santiago de Cali. Revista Científica Guillermo de Ockham, 8(1), 11-26. 
La informalidad urbana y los instrumentos de gestión del suelo

Autor: Alex Garcés Medrano

APA: Garcés-Medrano, A. (2010). La informalidad urbana y los instrumentos de gestión del suelo. Revista Científica Guillermo de Ockham, 8(1), 39-48.

Arbitramento voluntario Dificultades y oportunidades para su implementación en la solución alternativa de conflictos laborales. Aplicación en la jurisdicción laboral del circuito de Cali

Autores: Ángela María Bedoya Urrego, Iván Alberto Díaz Gutiérrez

APA: Bedoya-Urrego, A. M., \& Díaz-Gutiérrez, I. A. (2010). Arbitramento voluntario Dificultades y oportunidades para su implementación en la solución alternativa de conflictos laborales. Aplicación en la jurisdicción laboral del circuito de Cali. Revista Científica Guillermo de Ockham, 8(1), 49-63.

Second Life: una plataforma ideal para la química virtual

Autores: Katia María Noguera Oviedo, Jesús Olivero Verbel

APA: Noguera-Oviedo, K. M., \& Olivero-Verbel, J. (2010). Second Life: una plataforma ideal para la química virtual. Revista Científica Guillermo de Ockham, 8(1), 65-72.

Efecto de las condiciones de empacado y el tiempo de almacenamiento en el color del grano de fríjol seco cargamanto blanco (Phaseolus vulgaris L.)

Autor: Álex Fernando López Córdoba

APA: López-Córdoba, A. F. (2010). Efecto de las condiciones de empacadoy el tiempo de almacenamiento en el color del grano de fríjol seco cargamanto blanco (Phaseolus vulgaris L.). Revista Científica Guillermo de Ockham, 8(1), 65-72.

Caracterización fisicomecánica de concreto polimérico basado en resina poliéster

Autores: Mayra Valencia, Paola Collazos, José Mina, Edward Toro

APA: Valencia, M., Collazos, P., Mina, J., \& Toro E. (2010). Caracterización fisicomecánica de concreto polimérico basado en resina poliéster. Revista Científica Guillermo de Ockham, 8(1), 83-93.

Reflexiones sobre el proceso de modelado

Autores: Eliécer Pineda Ballesteros, Diana Patricia Landazábal

APA: Pineda-Ballesteros, E., \& Landazábal, D. P. (2010). Reflexiones sobre el proceso de modelado. Revista Científica Guillermo de Ockham, 8(1), 95-104.

Funciones parentales en familias recompuestas: nueve casos de mujeres de estrato socioeconómico bajo de Cali

Autor: María Elena Díaz Rico

APA: Díaz-Rico, M. E. (2010). Reflexiones sobre el proceso de modelado. Revista Científica Guillermo de Ockham, 8(1), 105-119.

Una lectura sobre deserción universitaria en estudiantes de pregrado desde la perspectiva de la minería de datos

Autor: Ricardo Timarán Pereira

APA: Timarán-Pereira, R. (2010). Una lectura sobre deserción universitaria en estudiantes de pregrado desde la perspectiva de la minería de datos. Revista Científica Guillermo de Ockham, 8(1), 121-130.

Pragmática y semiótica. Palabras, cosas, personas 
Autor: Sebastián Alejandro González Montero

APA: González-Montero, S. A. (2010). Una lectura sobre deserción universitaria en estudiantes de pregrado desde la perspectiva de la minería de datos. Revista Científica Guillermo de Ockham, 8(1), 131-144.

\section{Fronteras}

Naturaleza y tendencias de la ciencia, la tecnología y la innovación (CTI) en América Latina y el Caribe: un análisis crítico al modelo institucional colombiano.

Autor: Giovanni Carlos Argel Fuentes

APA: Argel-Fuentes, G. (2010). Naturaleza y tendencias de la ciencia, la tecnología y la innovación (CTI) en América Latina y el Caribe: un análisis crítico al modelo institucional colombiano. Revista Científica Guillermo de Ockham, 8(1), 147-163.

\section{Semblanzas}

Entrevista a Héctor Gallo

Autores: Manuel Alejandro Moreno y Aldemar Perdomo

APA: Moreno, M. A., \& Perdomo, A. (2010). Entrevista a Héctor Gallo. Revista Científica Guillermo de Ockham, 8(1), 167-181.

La condición humana: de la muerte y el suicidio. Una lectura de la obra de Albert Camus.

Autor: Edward Javier Ordoñez

APA: Ordoñez, E. J. (2010). La condición humana: de la muerte y el suicidio. Una lectura de la obra de Albert Camus. Revista Científica Guillermo de Ockham, 8(1), 183195.

\section{Reseña}

Libro: I/O Videojuegos, computadoras y seres humanos

Autor: Manuel Quiceno

APA: Quiceno, M. (2010). Reseña del libro I/O Videojuegos, computadoras y seres humanos, de C. M. Mejía \& M. Rodríguez-Mora. Revista Científica Guillermo de Ockham, 8(1), 199-200. 


\section{Vol. 8, Núm. 2 (2010)}

Tabla de contenidos

Artículos originales

Liderazgo moderno y tendencias gerenciales: cambios paradigmáticos en la gestión de la universidad como empresa del conocimiento

Autores: Wilmar Sierra Toncel, Ángela Ortiz Rojas, Olivia Rangel Luquez, Marelis Alvarado Mejía

APA: Sierra-Toncel, W., Ortiz-Rojas, A., Rangel-Luquez, O., \& Alvarado-Mejía, M. (2010). Liderazgo moderno y tendencias gerenciales: cambios paradigmáticos en la gestión de la universidad como empresa del conocimiento. Revista Científica Guillermo de Ockham, 8(2), 11-22.

El pensamiento estratégico Una integración de los sentidos con la razón

Autor: Olga Román Muñoz

APA: Román-Muñoz, O. (2010). El pensamiento estratégico Una integración de los sentidos con la razón. Revista Científica Guillermo de Ockham, 8(2), 23-36.

Incidencia de la logística en el marketing

Autores: Edy Lorena Burbano Vallejo, Rocío Morales Camacho

APA: Burbano-Vallejo, E., \& Morales-Camacho, R. (2010). Incidencia de la logística en el marketing. Revista Científica Guillermo de Ockham, 8(2), 37-54.

Factores psicosociales del ambiente laboral que caracterizan a funcionarios con contratación a término fijo e indefinido de una universidad privada de la ciudad de Cali

Autor: Octavio Alberto Orozco García

APA: Orozco-García, O. A. (2010). Factores psicosociales del ambiente laboral que caracterizan a funcionarios con contratación a término fijo e indefinido de una universidad privada de la ciudad de Cali. Revista Cientifica Guillermo de Ockham, $8(2), 55-68$.

La planificación del crecimiento urbano: entre la regulación, la flexibilización normativa y las desigualdades socio-espaciales

Autor: Cecilia Marengo

APA: Marengo, C. (2010). La planificación del crecimiento urbano: entre la regulación, la flexibilización normativa y las desigualdades socio-espaciales. Revista Científica Guillermo de Ockham, 8(2), 69-83.

Diálogos entre la articulación curricular y la formación investigativa

Autor: Orfa Garzón Rayo, Jessica Paola Gómez Álvarez

APA: Garzón-Rayo, O., \& Gómez-Álvarez, J. P. (2010). Diálogos entre la articulación curricular y la formación investigativa. Revista Científica Guillermo de Ockham, 8(2), 85-99.

Una comunidad virtual de apoyo a procesos de etnoeducación para la comunidad nasa del resguardo de Corinto Lópezadentro, departamento del Cauca - Colombia

Autores: Luz Marina Sierra Martínez, Roberto Carlos Naranjo Cuervo, Tulio Rojas Curieux 
APA: Sierra-Martínez, L. M., Naranjo-Cuervo, R. C., \& Rojas-Curieux, T. (2010). Una comunidad virtual de apoyo a procesos de etnoeducación para la comunidad nasa del resguardo de Corinto Lópezadentro, departamento del Cauca - Colombia. Revista Científica Guillermo de Ockham, 8(2), 101-114 .

Extracción y formulación de un bioinsecticida a partir de amida isobutílica 4,5-dihydropiperlonguminina extraída del pipilongo (Piper tuberculatum) para el control del pulgón amarillo (Sipha flava) en caña de azúcar (Sacharum officinarum)

Autores: Edwin Alberto López Devia, Jorge Durán Vanegas, Raúl Cuervo Mulet APA: López-Devia, E. A., Durán Vanegas, J., \& Cuervo-Mulet, R. (2010). Extracción y formulación de un bioinsecticida a partir de amida isobutílica 4,5-dihydropiperlonguminina extraída del pipilongo (Piper tuberculatum) para el control del pulgón amarillo (Sipha flava) en caña de azúcar (Sacharum officinarum). Revista Científica Guillermo de Ockham, 8(2), 115-123.

Revisión de la aritmética de curvas hiperelípticas para la implementación de un criptoprocesador a usarse en un sistema HECC

Autor: Oscar Casas García

APA: Casas-García, O. (2010). Revisión de la aritmética de curvas hiperelípticas para la implementación de un criptoprocesador a usarse en un sistema HECC. Revista Científica Guillermo de Ockham, 8(2), 125-139.

Efecto del número de capas en las propiedades superficiales de recubrimientos $\mathrm{Si} / \mathrm{Ti} / \mathrm{Zr}$ obtenidos a partir de suspensiones sol-gel

Autores: Jorge Hernando Bautista Ruiz, Gabriel Peña Rodríguez

APA: Bautista-Ruiz, J. H., \& Peña-Rodríguez, G. (2010). Efecto del número de capas en las propiedades superficiales de recubrimientos $\mathrm{Si} / \mathrm{Ti} / \mathrm{Zr}$ obtenidos a partir de suspensiones sol-gel. Revista Científica Guillermo de Ockham, 8(2), 141-148.

\section{Semblanza}

Entrevista a Jean Michel Vappereau

Autor: John Quintero

APA: Quintero, J. (2010). Entrevista a Jean Michel Vappereau. Revista Científica Guillermo de Ockham, 8(2), 151-162.

\section{Vol.9, Núm. 1 (2011)}

Tabla de contenidos

\section{Artículos originales}

Daños en puentes históricos de arco de ladrillo en el Alto Cauca (Colombia)

Autores: Jorge Galindo Díaz, Jairo Andrés Paredes López

APA: Galindo-Díaz, J., \& Paredes-López, J. A. (2011). Daños en puentes históricos de 
arco de ladrillo en el Alto Cauca (Colombia). Revista Científica Guillermo de Ockham, 9(1), 13-23.

Valores, disvalores o antivalores del cine colombiano (2000-2010).

Autor: Gloria Inés Ceballos Hurtado

APA: Ceballos-Hurtado, G. I. (2011). Valores, disvalores o antivalores del cine colombiano (2000-2010). Revista Científica Guillermo de Ockham, 9(1), 25-42.

El joven Hegel y la positividad de la religión cristiana

Autor: Carlos Andrés Méndez Sandoval

APA: Méndez-Sandoval, C. A. (2011). El joven Hegel y la positividad de la religión cristiana. Revista Científica Guillermo de Ockham, 9(1), 43-53.

Derecho penal de enemistad. Aproximaciones críticas al debate

Autores: Yesid Echeverry Enciso, Jefferson Jaramillo Marín

APA: Echeverry-Enciso, Y., \& Jaramillo-Marín, J. (2011). Derecho penal de enemistad. Aproximaciones críticas al debate. Revista Científica Guillermo de Ockham, 9(1), 55-79.

Resistencia de la yuca (manihot esculenta crantz) a la mosca blanca (aleurotrachelussocialis), mediante la tecnología del ADN recombinante

Autor: Raúl Cuervo, Jorge Durán

APA: Cuervo, R., \& Durán, J. (2011). Resistencia de la yuca (manihot esculenta crantz) a la mosca blanca (aleurotrachelussocialis), mediante la tecnología del ADN recombinante. Revista Científica Guillermo de Ockham, 9(1), 83-91.

Caracterización de recubrimientos a base de poliéster sobre AACLAD 2024-T3 mediante pruebas aceleradas de corrosión

Autores: William Aperador Chaparro, Andrés Felipe Escobar Mosquera, Jorge Hernando Bautista Ruiz

APA: Aperador-Chaparro, W., Escobar-Mosquera, A. F., \& Bautista-Ruiz, H. (2011). Caracterización de recubrimientos a base de poliéster sobre AACLAD 2024-T3 mediante pruebas aceleradas de corrosión. Revista Científica Guillermo de Ockham, 9(1), 93-101.

\section{Fronteras}

Notas para jugar con la ilusión biográfica y no perderse en el intento

Autor: Alexander Pereira Fernández

APA: Pereira-Fernández, A. (2011). Notas para jugar con la ilusión biográfica y no perderse en el intento. Revista Científica Guillermo de Ockham, 9(1), 105-122.

El sonido categorial de la salsa urbana

Autores: Jaime Villafañe y Guiovanny Lasso

APA: Villafañe, J., \& Lasso, G. (2011). El sonido categorial de la salsa urbana. Revista Científica Guillermo de Ockham, 9(1), 123-131. 
La educación social y popular en Colombia. Relaciones y búsquedas treinta años de legitimidad.

Autor: Claudia Vélez de la Calle.

APA: Vélez de la Calle, C. (2011). La educación social y popular en Colombia. Relaciones y búsquedas treinta años de legitimidad. Revista Científica Guillermo de Ockham, 9(1), 133-146.

\section{Semblanzas}

Una semblanza intelectual de Rodolfo de Roux

Autor: Helwar Figueroa

APA: Figueroa, H. (2011). Una semblanza intelectual de Rodolfo de Roux. Revista Científica Guillermo de Ockham, 9(1), 149-156.

Un maestro de la alegría: Fray Fernando Garzón Ramírez, Ofm

Autor: Beatriz Jaime Pérez

APA: Pérez, B. J. (2011). Un maestro de la alegría: Fray Fernando Garzón Ramírez, Ofm. Revista Científica Guillermo de Ockham, 9(1), 157-162.

\section{Reseñas}

Libro: Neopentecostalismo y política. El caso colombiano.

Autor: María Teresa Cifuentes Traslaviña

APA: Cifuentes-Traslaviña, M. T. (2011). Reseña del libro Neopentecostalismo y política. El caso colombiano, de A. Cepeda-Van Houten. Revista Científica Guillermo de Ockham, 9(1), 165-167.

Libro: El dosel sagrado. Para una teoría sociológica de la religión.

Autor: Luis Alberto Valderrama

APA: Valderrama, L. A. (2011). Reseña del libro El dosel sagrado. Para una teoría sociológica de la religión, de P. Berger. Revista Científica Guillermo de Ockham, 9(1), 169-171.

Libro: Between Tyranny and Anarchy: a History of democracy in Latin America, 1800-2006

Autor: Juan Carlos Sánchez Sierra

APA: Sánchez-Sierra, J. C. (2011). Reseña del libro Between Tyranny and Anarchy: a History of democracy in Latin America, 1800-2006, de P. W. Drake. Revista Cientifica Guillermo de Ockham, 9(1), 173-176.

libro: Vamos a mediar

Autor: Rebecca R. Rutemberg

APA: Rutemberg, R. R. (2011). Reseña del libro Vamos a mediar, de S. Lerer. Revista Científica Guillermo de Ockham, 9(1), 177-178. 


\section{Vol. 9, Núm. 2(2011)}

Tabla de contenidos

\section{Artículos originales}

Educabilidad, formación y antropología pedagógica: repensar la educabilidad a la luz de la tradición pedagógica alemana

Autores: Andrés Klaus Runge Peña, Juan Felipe Garcés Gómez

APA: Runge-Peña, A. K., \& Garcés-Gómez, J. F. (2011). Educabilidad, formación y antropología pedagógica: repensar la educabilidad a la luz de la tradición pedagógica alemana. Revista Científica Guillermo de Ockham, 9(2), 13-25.

El caso de las mujeres inmigrantes colombianas en Majadahonda (España) vinculadas al servicio doméstico

Autor: Sandra Lorena Botina Narváez

APA: Botina-Narváez, S. L. (2011). El caso de las mujeres inmigrantes colombianas en Majadahonda (España) vinculadas al servicio doméstico. Revista Científica Guillermo de Ockham, 9(2), 27-40.

Discursos y estrategias de la Iglesia Católica frente a la pluralización religiosa en Colombia Autor: Cristina Moreno

APA: Moreno, C. (2011). Discursos y estrategias de la Iglesia Católica frente a la pluralización religiosa en Colombia. Revista Científica Guillermo de Ockham, 9(2), 41-51.

El Alterius como principio para la ética de la alteridad

Autor: William Rojas Cordero

APA: Rojas-Cordero, W. (2011). El Alterius como principio para la ética de la alteridad. Revista Científica Guillermo de Ockham, 9(2), 53-59.

El mensaje de Jesús y el mensaje de Pablo desde una lectura no teológica del Nuevo Testamento

Autor: Luis Alberto Valderrama Valderrama

APA: Valderrama-Valderrama, L. A. (2011). El mensaje de Jesús y el mensaje de Pablo desde una lectura no teológica del Nuevo Testamento. Revista Científica Guillermo de Ockham, 9(2), 61-76.

Evaluación de la calidad del agua en la Ciénaga de la Virgen (Cartagena, Colombia) durante el período 2006-2010

Autores: Wilson Maldonado, Ildefonso Baldiris, Jhon Díaz

APA: Maldonado, W., Baldiris, I., \& Díaz, J. (2011). Evaluación de la calidad del agua en la Ciénaga de la Virgen (Cartagena, Colombia) durante el período 2006-2010. Revista Cientifica Guillermo de Ockham, 9(2), 79-87.

Validación de un método ágil para el análisis de riesgos de la información digital 
Autores: Luis Merchán Paredes, Diego Gómez Mosquera

APA: Merchán-Paredes, L., \& Gómez-Mosquera, D. (2011). Validación de un método ágil para el análisis de riesgos de la información digital. Revista Científica Guillermo de Ockham, 9(2), 89-100.

\section{Fronteras}

Corpo-empresa na industria do espectáculo

Autor: Debora Tabacof

APA: Tabacof, D. (2011). Corpo-empresa na industria do espectáculo. Revista Científica Guillermo de Ockham, 9(2), 103-107.

\section{Semblanzas}

Esclavitud y libertad en el Valle de Río Cauca

Autor: Michael Taussig y Anna Rubbo

APA: Taussig, M., \& Rubbo, A. (2011). Esclavitud y libertad en el Valle de Río Cauca. Revista Científica Guillermo de Ockham, 9(2), 111-118.

\section{Reseñas}

Libro: La deconstrucción y reconstrucción del sujeto jurídico fe menino. Una reflexión práctica para el ejercicio del derecho.

Autor: Liliana Castillo

APA: Castillo, L. (2011). Reseña del libro La deconstrucción y reconstrucción del sujeto jurídico femenino. Una reflexión práctica para el ejercicio del derecho, de D. M. Bustamante-Arango \& L. Ambuila-Valencia. Revista Científica Guillermo de Ockham, 9(2), 121-124.

Libro: Ciudadanos de dos mundos. El movimiento evangélico

\section{Autor: Marcos Carbonelli}

APA: Carbonelli, M. (2011). Reseña del libro Ciudadanos de dos mundos. El movimiento evangélico, de H. Wynarczyk. Revista Científica Guillermo de Ockham, 9(2), 125-127.

Libro: El gran fraude ¿violencia antisindical en Colombia? Historia de la conspiración contra el TLC.

Autor: Alexander Pereira Fernández

APA: Pereira-Fernández, A. (2011). Reseña del libro El gran fraude ¿iviolencia antisindical en Colombia? Historia de la conspiración contra el TLC, de L. BoteroCampuzano. Revista Científica Guillermo de Ockham, 9(2), 125-127.

Libro: Tirándole libros a las balas. Memoria de la violencia antisindical contra educadores, 1978-2008.

Autor: AlexanderPereira Fernández

APA: Pereira-Fernández, A. (2011). Reseña del libro Tirándole libros a las balas. Memoria de la violencia antisindical contra educadores, 1978-2008, de G.

Correa \& J. D. González. Revista Científica Guillermo de Ockham, 9(2), 135-138. 
Libro: Justicia cordial

Autor: Edward Javier Ordoñez

APA: Ordoñez, E. J. (2011). Reseña del libro Justicia cordial, de A. Cortina. Revista Científica Guillermo de Ockham, 9(2), 139-143.

Libro: Sobre ideología y democracia en Argentina y Brasil entre 1980-2003

Autor: Nicolás Damin

APA: Damin, N. (2011). Reseña del libro Sobre ideología y democracia en Argentina y Brasil entre 1980-2003, de A. Salas-Oroño. Revista Científica Guillermo de Ockham, 9(2), 145-147.

\section{Vol. 10, Núm. 1 (2012)}

Tabla de contenidos

\section{Artículos originales}

Juventud y protesta global hoy: por un análisis retrospectivo

Autor: Álvaro Acevedo Tarazona, Gabriel Samacá Alonso

APA: Acevedo-Tarazona, A., \& Samacá-Alonso, G. (2012). Juventud y protesta global hoy: por un análisis retrospectivo. Revista Científica Guillermo de Ockham, 10(1), 15-25.

Territorios, internacionalización y desarrollo. Aproximación teórica a una realidad evidente

Autor: William Guillermo Jiménez

APA: Guillermo-Jiménez, W. (2012). Territorios, internacionalización y desarrollo. Aproximación teórica a una realidad evidente. Revista Científica Guillermo de Ockham, 10(1), 15-25

Evangélicos y política: tensiones en torno a la libertad e igualdad religiosa en Argentina

Autores: Marcos Carbonelli, Mariela Mosqueira

APA: Carbonelli, M., \& Mosqueira, M. (2012). Evangélicos y política: tensiones en torno a la libertad e igualdad religiosa en Argentina. Revista Científica Guillermo de Ockham, 10(1), 27-40.

Antagonismo y participación en la Constitución de 1991

Autor: John Fredy Hoyos

APA: Hoyos, J. F. (2012). Antagonismo y participación en la Constitución de 1991. Revista Científica Guillermo de Ockham, 10(1), 41-54.

La vigencia kantiana del concepto puro del derecho para el caso colombiano: hacia una paz democrática estable

Autor: William Farfán Moreno

APA: Farfán-Moreno, W. (2012). La vigencia kantiana del concepto puro del derecho para el caso colombiano: hacia una paz democrática estable. Revista Científica Guillermo de Ockham, 10(1), 67-81. 
Anatomía del conflicto armado en el Valle del Cauca durante la primera década del siglo XXI

Autor: Catalina Acosta Oidor

APA: Acosta-Oidor, C. (2012). Anatomía del conflicto armado en el Valle del Cauca durante la primera década del siglo XXI. Revista Científica Guillermo de Ockham, 10(1), 83-99.

Diseño de compensadores para una planta que presenta ciclo de histéresis, tiempo de retardoy altas variaciones en sus parámetros.

Autores: Carlos Héctor Cruz Vergara, Rubén Darío Nieto Londoño y Jesús Alfonso López Sotelo

APA: Cruz-Vergara, C. H., Nieto-Londoño, R. D., \& López-Sotelo, J. A. (2012). Diseño de compensadores para una planta que presenta ciclo de histéresis, tiempo de retardo y altas variaciones en sus parámetros. Revista Científica Guillermo de Ockham, 10(1), 103-113.

\section{Fronteras}

Traición se escribe con "efe". Un ensayo sobre la academia y sus angustiadores

Autores: Ramiro Ceballos

APA: Ceballos, R. (2012). Traición se escribe con "efe". Un ensayo sobre la academia y sus angustiadores. Revista Científica Guillermo de Ockham, 10(1), 117122.

Libertad religiosa y educación en Colombia: Ni intocables ni míticas

Autor: Alberto Echeverri

APA: Echeverri, A. (2012). Libertad religiosa y educación en Colombia: Ni intocables ni míticas. Revista Científica Guillermo de Ockham, 10(1), 123-134.

O corpo e os ideas no malestar femenino

Autor: Maria Helena Fernandez

APA: Fernandez, M. H. (2012). O corpo e os ideas no malestar femenino. Revista Científica Guillermo de Ockham, 10(1), 135-140.

\section{Se mblanza}

Fabián: un libro abierto, un maestroy un amigo.

Autor: Luis Humberto Cardona Artunduaga

APA: Cardona-Artunduaga, L. H. (2012). Fabián: un libro abierto, un maestroy un amigo. Revista Científica Guillermo de Ockham, 10(1), 135-140.

\section{Vol. 10. Núm. (2) \\ Tabla de contenidos \\ Artículos originales}

Mundos populares entre el desplazamiento y el poblamiento Autores: Jesús Darío González Bolaños

APA: González, J. D. (2012). Mundos populares entre el desplazamiento y el 
poblamiento. Revista Científica Guillermo de Ockham, 10(2), 13-28.

Corporalidad religiosa: el gesto como construcción de identidad

Autor: David Avilés Aguirre

APA: Avilés, D. (2012). Corporalidad religiosa: el gesto como construcción de identidad. Revista Científica Guillermo de Ockham, 10(2), 29-36.

Procesos de formación de líderes investigadores

Autor: Lina María Zuluaga Giraldo, Jemay Mosquera Téllez, Mercedes Higuera Peña

APA: Zuluaga, L. M., Mosquera, J., \& Higuera, M. (2012). Procesos de formación de líderes investigadores. Revista Científica Guillermo de Ockham, 10(2), 37-47.

Tipos de aprendizaje promovidos por los profesores de matemática y ciencias naturales del sector oficial del departamento del Quindío, Colombia.

Autor: Ana Patricia León Urquijo, Liliana Patricia Ospina Marulanda, Robinson Ruiz Lozano

APA: León-Urgijo, A. P., Ospina, L., Ruiz, R. (2012). Tipos de aprendizaje promovidos por los profesores de matemática y ciencias naturales del sector oficial del departamento del Quindío, Colombia. Revista Científica Guillermo de Ockham, 10(2), 49-63.

Ética: directriz para toma de decisiones en universidades

Autor: Marelis Alvarado Mejía, Olivia Rangel Luquéz

APA: Alvarado, M., \& Rangel, O. (2012). Ética: directriz para toma de decisiones en universidades. Revista Científica Guillermo de Ockham, 10(2), 65-74.

El contenido del numeral 1엉 del artículo 230 del CPP frente al bloque de constitucionalidad

Autor: José Freddy Restrepo García

APA: Restrepo, J. F. (2012). El contenido del numeral 10 del artículo 230 del CPP frente al bloque de constitucionalidad. Revista Científica Guillermo de Ockham, 10(2), 75-89.

Modelo matemático y herramienta de simulación de exoesqueleto activo de cinco segmentos

Autor: Juan Carlos Cruz Ardila, José Miguel Ramírez Escarpeta

APA: Cruz, J. C., \& Ramírez, J. M. (2012). Modelo matemático y herramienta de simulación de Gexoesqueleto activo de cinco segmentos. Revista Científica Guillermo de Ockham, 10(2), 93-105.

\section{Fronteras}

Una mirada a la metamorfosis religiosa en América Latina: nuevas ofertas de sentido en la sociedad contemporánea.

Autor: Luis Andrés Bahamondes González.

APA: Bahamondes, L. A. (2012). Una mirada a la metamorfosis religiosa en América Latina: nuevas ofertas de sentido en la sociedad contemporánea. Revista Científica Guillermo de Ockham, 10(2), 93-108.

Relatos de poesía divina y humana afrodescendiente: rituales de limpieza y nacimiento.

Autores: Rosa Patricia Quintero

APA: Quintero, R. P. (2012). Relatos de poesía divina y humana afrodescendiente: 
rituales de limpieza y nacimiento. Revista Científica Guillermo de Ockham, 10(2), 117-128.

La importancia de la tradición oral: El grupo Coyaima-Colombia

Autor: Nancy Ramirez Poloche

APA: Ramírez, N. (2012). La importancia de la tradición oral: El grupo Coyaima-

Colombia. Revista Científica Guillermo de Ockham, 10(2), 129-144.

O corpo enfermo e a morte como dádivas: uma análise da antropología do dom no filme Gritos a sussurros de Igmar Bergman.

Autor: Edilson Baltazar Barreira

APA: Baltazar, E. (2012). O corpo enfermo e a morte como dádivas: uma análise da antropología do dom no filme Gritos a sussurros de Igmar Bergman. Revista Científica Guillermo de Ockham, 10(2), 145-152.

Novas formas organizacionais e o assédio moral no trabalho

Autor: Ladislau Ribeiro do Nacimento

APA: Ribeiro, L. (2012). Novas formas organizacionais e o assédio moral no trabalho. Revista Científica Guillermo de Ockham, 10(2), 153-160.

La novia árabe de Daniela Jazame

Autor: Jury Amira del Valle

APA: Amira del Valle, J. (2012). La novia árabe de Daniela Jazame. Revista

Científica Guillermo de Ockham, 10(2), 161-164.

\section{Semblanzas}

Jairo Varela Martínez. No murió un hombre, no murió un músico, murió una época.

Autor: Jaime Villafañe Padilla

APA: Villafañe, J. (2012). Jairo Varela Martínez. No murió un hombre, no murió un

\section{Reseñas}

músico, murió una época. Revista Científica Guillermo de Ockham, 10(2), 175-189.

Basta de historias la observación latinoamericana con el pasado y las doce claves del futuro.

Autor: Edgar Alonso Vanegas

APA: Vanegas, E. (2012). Reseña del libro Basta de historias la observación latinoamericana con el pasado y las doce claves del futuro, de A. Oppenheimer.

Revista Científica Guillermo de Ockham, 10(2), 193-212.

Maestra vida. Relatos de la parecía en la ciudad popular.

Autor: Helwar Figueroa Salamanca

APA: Figueroa, H. (2012). Reseña del libro Maestra vida. Relatos de la parecía en la ciudad popular, de J. D. González. Revista Científica Guillermo de Ockham, 10(2), 213-215.

Agroindustrialización de la carne de cuy

Autor: Mauricio Quevedo Pantoja

APA: Quevedo, M. (2012). Reseña del libro Agroindustrialización de la carne de cuy, de F. E. Argote \& R. A. Cuervo. Revista Científica Guillermo de Ockham, 10(2), 217-218.

Patronando emociones.

Autor: Elezabeth Vejarano Soto 
APA: Vejarano, E. (2012). Reseña del libro Patronando emociones, de C. I. Serna. Revista Científica Guillermo de Ockham, 10(2), 219-220.

Fundamentos epistemológicos de las psicologías. Con énfasis en psicología transpersonal Autor: Carlos Calle

APA: Calle, C. (2012). Reseña del libro Fundamentos epistemológicos de las psicologías. Con énfasis en psicología transpersonal, de J. Duque, P. Lasso \& J. Orejuela. Revista Científica Guillermo de Ockham, 10(2), 221-222.

Vol.11Núm. (1)

Tabla de contenidos

Artículos originales

Una fractura desde el feminismo árabe: a propósito de Fatema Mernissi

Autor: Edward Javier Ordóñez

APA: Ordóñez, E. J. (2013). Una fractura desde el feminismo árabe: a propósito de Fatema Mernissi. Revista Científica Guillermo de Ockham, 11(1), 11-19.

Autonomia, saúde mental e subjetividade no contexto assistencial brasileiro

Autor: Daniel Magalhães Goulart

APA: Magalhães, D. (2013). Autonomia, saúde mental e subjetividade no contexto assistencial brasileiro. Revista Científica Guillermo de Ockham, 11(1), 21-33.

El edificio para el Batallón Pichincha de Cali (1906-1968)

Autor: Jorge Galindo Díaz, Laura María Henao Montoya

APA: Galindo, J., \& Henao, L. M. (2013). El edificio para el Batallón Pichincha de

Cali (1906-1968). Revista Científica Guillermo de Ockham, 11(1), 33-47.

Comisaría de Familia de Canapote: entidad que genera capital social.

Autor: Judith Eugenia Herrera Hernández, Camilo Alberto Madariaga Orozco

APA: Herrera, J., \& Madariaga, A. (2013). Comisaría de Familia de Canapote: entidad que genera capital social. Revista Científica Guillermo de Ockham, 11(1), 49-64.

Modelado, simulación e implementación de controladores LQR y RLVE al sistema péndulo invertido rotacional usando la plataforma NI ELVIS II.

Autor: Álvaro Romero Acero, Alejandro Marín Cano, Jovani Alberto Jiménez Builes. APA: Romero, A., Marín, A., \& Jiménez, J. (2013). Modelado, simulación e implementación de controladores LQR y RLVE al sistema péndulo invertido rotacional usando la plataforma NI ELVISII. Revista Científica Guillermo de Ockham, 11(1), 67-78.

Deshidrogenación catalítica del etilbenceno a estireno

Autor: Robert Causado Escobar, Rosario Muñoz Martínez, Aída Liliana Barbosa López

APA: Causado, R. E., Muñoz, R., \& Barbosa, A. (2013). Deshidrogenación catalítica del etilbenceno a estireno. Revista Científica Guillermo de Ockham, 11(1), 79-95.

Producción y procesamiento del maíz en Colombia

Autor: Carlos David Grande Tovar, Brigitte Sthepani Orozco Colonia

APA: Grande, C., \& Orozco, B. (2013). Producción y procesamiento del maíz en 
Colombia. Revista Científica Guillermo de Ockham, 11(1), 97-110.

Fuentes críticas de resistencia aerodinámica en un tren urbano de mediana distancia: un enfoque CFD

Autor: Andrés Tabares, Natalia Gómez, César Nieto, Mauricio Giraldo

APA: Tabares, A., Gómez, N., Nieto, C., \& Giraldo, M. (2013). Fuentes críticas de resistencia aerodinámica en un tren urbano de mediana distancia: un enfoque CFD.

Revista Científica Guillermo de Ockham, 11(1), 111-124.

Nanotecnología aplicada a la medicina

Autor: Nerlis Pájaro Castro, Jesús Olivero Verbel, Juan Redondo Padilla

APA: Castro, N., Olivero, J., \& Redondo, J. (2013). Nanotecnología aplicada a la medicina. Revista Científica Guillermo de Ockham, 11(1), 125-133.

\section{Fronteras}

La encíclica apócrifa de León XIII: Un episodio del anticlericalismo latinoamericano

Autor: Gerardo Alberto Hernández

APA: Hernández, G. A. (2013). La encíclica apócrifa de León XIII: Un episodio del anticlericalismo latinoamericano. Revista Científica Guillermo de Ockham, 11(1), 137-148.

El mito del primer mundo. Capitalismo y barbarie.

Autores: Nancy Ramírez

APA: Ramírez, N. (2013). El mito del primer mundo. Capitalismo y barbarie. Revista Científica Guillermo de Ockham, 11(1), 149-162.

¿Liquidar la educación o educación liquida?

Autor: Guillermo Bustamante

APA: Bustamante, G. (2013). ¿Liquidar la educación o educación liquida?. Revista Cientifica Guillermo de Ockham, 11(1), 163-170.

\section{Semblanzas}

Apuntes para una semblanza intelectual de Jesús Martín Barbero

Autores: Beatriz Jaime Pérez

APA: Pérez, B. (2013). Apuntes para una semblanza intelectual de Jesús Martín Barbero. Revista Científica Guillermo de Ockham, 11(1), 173-178.

\section{Reseñas}

Yo maté a Sherezade. Confesiones de una mujer árabe furiosa.

Autor: Edward Javier Ordoñez, Iván Enrique Valdez

APA: Ordoñez, J., \& Valdez, I. (2011). Reseña del libro Yo maté a Sherezade. Confesiones de una mujer árabe furiosa, de J. Haddad. Revista Científica Guillermo de Ockham, 11(1), 181-183.

Tinta indeleble. Guillermo Cano vida y obra

Autor: Diana Ximena Bejarano

APA: Bejarano, D. (2013). Reseña del libro Tinta indeleble. Guillermo Cano vida y obra, de M. Cano, J. Cardona, C. Correa, M. Vallejo. Revista Científica Guillermo de Ockham, 11(1), 185-187.

Palabra plena. Conversaciones con psicoanálisis

Autor: Alejandra Ordoñez

APA: Ordoñez, A. (2013). Reseña del libro Palabra plena. Conversaciones con 
psicoanálisis, de J. Orejuela. Revista Científica Guillermo de Ockham, 11(1), 189-192.

Gramáticas actuales de la relación hombre-trabajo. Propuestas de lectura.

Autor: Andrés Caballero

APA: Caballero, A. (2013). Reseña del libro Gramáticas actuales de la relación hombre-trabajo. Propuestas de lectura, de S. Malvezzi, J. Orejuela, R. Marcus, J. Vesga \& W. Riascos. Revista Científica Guillermo de Ockham, 11(1), 193-194.

\section{Vol. 11, Núm. (2)}

Artículos originales

Telenovelas, narcotráfico y conciencia política en Latinoamérica. Perspectivas sobre un problema de estudio. Telenovelas, narcotráfico y conciencia política en Latinoamérica. Perspectivas sobre un problema de estudio

Autor: Juan Carlos Sánchez Sierra

APA: Sánchez-Sierra, J. C. (2013). Telenovelas, narcotráfico y conciencia política en Latinoamérica. Perspectivas sobre un problema de estudio. Telenovelas, narcotráfico y conciencia política en Latinoamérica. Perspectivas sobre un problema de estudio. Revista Científica Guillermo de Ockham, 11(2), 15-33.

Cuando se vive el desarraigo. Educación y desplazamiento forzado: una mirada desde el Distrito de Aguablanca, Cali, Colombia

Autor: Patricia Lasso Toro

APA: Lasso, P. (2013). Cuando se vive el desarraigo. Educación y desplazamiento forzado: una mirada desde el Distrito de Aguablanca, Cali, Colombia. Revista Cientifica Guillermo de Ockham, 11(2), 35-51.

Imaginarios sociopolíticos de los jóvenes indígenas en la ciudad de Bogotá

Autor: Cristhian Uribe Mendoza

APA: Uribe-Mendoza, C. (2013). Imaginarios sociopolíticos de los jóvenes indígenas en la ciudad de Bogotá. Revista Científica Guillermo de Ockham, 11(2), 53-67.

El sistema de responsabilidad penal para adolescentes: la expansión de la punibilidad en el neopunitivismo colombiano.

Autor: Omar Huertas Díaz, Iván Ricardo Morales Chinome

APA: Huertas, O., \& Morales, D. R. (2013). El sistema de responsabilidad penal para adolescentes: la expansión de la punibilidad en el neopunitivismo colombiano.

Revista Científica Guillermo de Ockham, 11(2), 69-78.

Una mirada a la responsabilidad civil española: el régimen subjetivo

Autor: Rubén Acevedo Prada

APA: Acevedo, R. (2013). Una mirada a la responsabilidad civil española: el régimen subjetivo. Revista Científica Guillermo de Ockham, 11(2), 79-88.

Cultura organizacional y sistemas de gestión de la calidad: una relación clave en la gestión de las instituciones de educación superior

Autor: Juan Javier Vesga

APA: Vesga, J. J. (2013). Cultura organizacionaly sistemas de gestión de la calidad: una relación clave en la gestión de las instituciones de educación superior. Revista Científica Guillermo de Ockham, 11(2), 89-100.

Construcción de argumentos durante la producción de textos digitales 
Autor: Gerzon Yaír Calle Álvarez

APA: Calle-Álvarez, G. Y. (2013). Construcción de argumentos durante la producción de textos digitales. Revista Científica Guillermo de Ockham, 11(2), 101-114.

\section{Fronteras}

Tradición y palabra: el santo oficio de la memoria (la historia de vida y el método biográfico)

Autor: Álvaro Acevedo Tazona

APA: Tarazona, A. (2013). Tradición y palabra: el santo oficio de la memoria (la historia de vida y el método biográfico). Revista Científica Guillermo de Ockham, $11(2), 137-148$.

Reflexiones en torno a la didáctica de la historia.

Autor: Aceneth Perafán Cabrera

APA: Perafán, A. (2013). Reflexiones en torno a la didáctica de la historia. Revista Científica Guillermo de Ockham, 11(2), 149-160.

Empatía y alteridad en Mariatégui: una lectura de los siete ensayos.

Autor: Edwin Cruz-Rodríguez

APA: Cruz-Rodríguez, E. (2013). Empatía y alteridad en Mariatégui: una lectura de los siete ensayos. Revista Científica Guillermo de Ockham, 11(2), 161-172.

Peter sloterdijk: pensar al hombre en una época post-humanista.

Autor: Carlos Andrés Méndez

APA: Méndez, C. A. (2013). Peter sloterdijk: pensar al hombre en una época posthumanista. Revista Científica Guillermo de Ockham, 11(2), 173-186.

El hombre sin el ser: implicación antropológica del pensamiento de Jean-Luc Marion

Autor: Wilfer Alexis Yepes Muñoz

APA: Yepes, W. A. (2013). El hombre sin el ser: implicación antropológica del pensamiento de Jean-Luc Marion. Revista Científica Guillermo de Ockham, 11(2), 187-196.

Investigación de mercado sobre el grado de aceptación de mermelada de cocona en sibundoy, Putumayo

Autores: Francisco Emilio Argote, Diana Patricia Vargas, Hector Samuel Villada APA: Argote, F., Vargas, D., \& Villada, H. (2013). Investigación de mercado sobre el grado de aceptación de mermelada de cocona en sibundoy, Putumayo. Revista Científica Guillermo de Ockham, 11(2), 197-205.

\section{Semblanza}

Entrevista a Jean Michael Vappereau

Autor: Johnny Javier Orejuela, Jhon Alexander Quintero

APA: Orejuela, J., \& Quintero, J. (2013). Entrevista a Jean Michael Vappereau. Revista Científica Guillermo de Ockham, 11(2), 209-219.

\section{Reseña}

Identidades colectivas y reconocimiento razas, etnias, géneros y sexualidades

Autor: Laura Silva Chica 
APA: Silva, L. (2013). Reseña del libro Identidades colectivas y reconocimiento razas, etnias, géneros y sexualidades, de D. I. Grueso \& G. Castellanos. Revista Científica Guillermo de Ockham, 11(2), 223-227.

El cisne negro. El impacto de lo altamente improbable

Autor: Diana Ximena Bejarano

APA: Bejarano, D. (2013). Reseña del libro El cisne negro. El impacto de lo altamente improbable, de N. Taleb. Revista Científica Guillermo de Ockham, 11(2), 229-232.

El management no es como lo pintan. Esbozo de hipótesis sobre algunas condiciones que posibilitaron la emergencia del modo de ser management.

Autor: Carlos Alberto Molina Gómez

APA: Molina, C. (2013). Reseña del libro El management no es como lo pintan. Esbozo de hipótesis sobre algunas condiciones que posibilitaron la emergencia del modo de ser management, de C, Molina. Revista Científica Guillermo de Ockham, 11(2), 237-241.

La contracultura juvenil. De la emancipación a los indignados

Autor: Edward Javier Ordoñez, Mario Leal Betancourt

APA: Ordoñez, E., \& Leal, M. (2013). Reseña del libro La contracultura juvenil. De la emancipación a los indignados, de Revista Científica Guillermo de Ockham, 11(2), 243-245.

\section{Vol. 12, Núm. 1 (2014)}

Tabla de contenidos

Artículos originales

Cognición de grupo y cognición de enjambre en la solución del problema del agente viajero

Autor: Juan Felipe Martínez

APA: Martínez, J. F. (2014). Cognición de grupo y cognición de enjambre en la solución del problema del agente viajero. Revista Científica Guillermo de Ockham, 12(1), 9-18.

Nociones de niños de ocho a diez años acerca de la justicia en las sanciones escolares Autores: John Aníbal Gómez Varón, Diana Patricia Londoño Serna, María Victoria Montañez Gómez

APA: Gómez-Varón, J. A., Londoño-Serna, D. P., \& Montañez-Gómez, M. V. (2014). Nociones de niños de ocho a diez años acerca de la justicia en las sanciones escolares. Revista Científica Guillermo de Ockham, 12(1), 19-28.

Prácticas educativas de una maestra de transición. Un estudio sociocultural constructivista

Autores: Mónica Roncancio Moreno, Angela Branco

APA: Roncancio-Moreno, M., \& Branco, A. (2014). Prácticas educativas de una maestra de transición. Un estudio sociocultural constructivista. Revista Científica Guillermo de Ockham, 12(1), 29-34. 
Oportunidades, sostenibilidad y riesgos de los negocios informales de la comuna 10 de Cali

Autores: Julio César Ramírez Reyes, Gerardo Alexander Vergara Mesa

APA: Ramírez-Reyes, J. C., \& Vergara-Mesa, G. A. (2014). Oportunidades, sostenibilidad y riesgos de los negocios informales de la comuna 10 de Cali. Revista Científica Guillermo de Ockham, 12(1), 35-42.

Evaluating interactive systems from an emotional perspective Autores: Yenny Alexanra Méndez Alegria, Cesar Alberto Collazos Ordóñez, Antoni Granollers Saltiveri, Alfredo Sanchez Huitr

APA: Méndez-Alegria, Y. A., Collazos-Ordóñez, C. A., Granollers-Saltiveri, A., \& Sanchez Huitr, A. (2014). Evaluating interactive systems from an emotional perspective. Revista Científica Guillermo de Ockham, 12(1), 43-50.

La juventud campesina en los programas de Acción Cultural Popular

Autor: Nurys Esperanza Silva Cantillo

APA: Silva-Cantillo, N. E. (2014). La juventud campesina en los programas de Acción Cultural Popular. Revista Científica Guillermo de Ockham, 12(1), 51-64.

Parrhesia: Entre ley y filosofía

Autor: Víctor Hugo Vásquez Gómez

APA: Vásquez-Gómez, V. H. (2014). Parrhesia: Entre ley y filosofía. Revista Científica Guillermo de Ockham, 12(1), 65-76.

Tensiones del sujeto entre lo instituido y lo instituyente. Del Frente Nacional a la Constitución de 1991

Autor: Edgar Fernández Fonseca

APA: Fernández-Fonseca, E. (2014). Tensiones del sujeto entre lo instituido y lo instituyente. Del Frente Nacional a la Constitución de 1991. Revista Científica Guillermo de Ockham, 12(1), 77-92.

\section{Fronteras :}

Rasgos de la ontología fundamental

Autor: Edward Javier Ordóñez

APA: Ordoñez, E. J. (2014). Rasgos de la ontología fundamental. Revista Científica Guillermo de Ockham, 12(1), 95-102.

\section{Se mblanzas :}

No tengo muchos discos que mostrar, pero sí muchas historias para contar

Autor: Johnny Javier Orejuela

APA: Orejuela, J. J. (2014). No tengo muchos discos que mostrar, pero sí muchas historias para contar. Revista Científica Guillermo de Ockham, 12(1), 105-110.

Paz no es ausencia de conflicto: Es desarrollo y seguridad

Autor: Silvio Lerer

APA: Lerer, S. (2014). Paz no es ausencia de conflicto: Es desarrollo y seguridad. Revista Científica Guillermo de Ockham, 12(1), 111-113. 


\section{Reseñas :}

Libro: Pulgarcita. "El mundo cambió tanto que los jóvenes deben reinventar todo: una manera de vivir juntos, instituciones, una manera de ser y de conocer"

Autor: Jean Nikola Cudina

APA: Cudina, J. N. (2014). Reseña del libro Pulgarcita. “El mundo cambió tanto que los jóvenes deben reinventar todo: una manera de vivir juntos, instituciones, una manera de ser y de conocer", de M. Serres. Revista Científica Guillermo de Ockham, 12(1), 117-118.

Libro: Floralba Cano Escritos

Autor: Carlos Alfonso Calle

APA: Calle, C. A. (2014). Reseña del libro Floralba Cano Escritos, de J. J. Orejuela. Revista Cientifica Guillermo de Ockham, 12(1), 119-122.

Por qué fracasan los países. Los orígenes del poder, la prosperidad y la pobreza

Autor: Francisco Javier Pantoja Pantoja

APA: Pantoja-Pantoja, F. J. (2014). Reseña del libro Por qué fracasan los países. Los orígenes del poder, la prosperidad y la pobreza, de D. Acemoglu \& J. A. Robinson. Revista Científica Guillermo de Ockham, 12(1), 123-125. 